\title{
Effect of Daikenchuto (TJ-100) on gastrointestinal symptoms following laparoscopic colectomy in patients with colon cancer: study protocol for a randomized controlled trial
}

Nobuaki Hoshino, Kenji Kawada*, Koya Hida, Toshiaki Wada, Ryo Takahashi, Mami Yoshitomi and Yoshiharu Sakai

\begin{abstract}
Background: Postoperative paralytic ileus can be a difficult complication for both surgeons and patients. Causes and treatments have been discussed for more than two centuries, but have not yet been fully resolved.

Daikenchuto (TJ-100, DKT) is a traditional Japanese herbal medicine. Recently, some beneficial mechanisms of DKT to relieve paralytic ileus have been reported. DKT can suppress inflammation, increase intestinal blood flow, and accelerate bowel movements. Therefore, we have designed a randomized controlled trial to investigate the effects of DKT on postoperative gastrointestinal symptoms following laparoscopic colectomy in patients with left-sided colon cancer at a single institution.
\end{abstract}

Methods/design: As primary endpoints, the following outcomes will be evaluated: (i) grade of abdominal pain determined using the numeric rating scale (NRS), (ii) grade of abdominal distention determined using the NRS, and (iii) quality of life determined using the Gastrointestinal Quality Life Index (GIQLI). As secondary endpoints, the following will be evaluated: (i) postoperative nutritional status (Onodera's Prognostic Nutritional Index (PNI) and the Controlling Nutritional Status score (CONUT score)), (ii) duration to initial flatus, (iii) duration to initial defecation, (iv) bowel gas volume, (v) character of stool (Bristol Stool Form Scale), (vi) defecation frequency per day, (vii) postoperative complications (Clavien-Dindo classification), (viii) length of postoperative hospital stay, and (ix) metabolites in the stool and blood. This trial is an open-label study, and needs to include 40 patients (20 patients per group) and is expected to span 2 years.

Discussion: To our knowledge, this is the first randomized controlled trial to investigate the effects of DKT on postoperative subjective outcomes (i.e., postoperative quality of life) following laparoscopic colectomy as primary endpoints. Exploratory metabolomics analysis of metabolites in stool and blood will be conducted in this trial, which previously has only been performed in a few human studies. The study aims to guide a future full-scale pragmatic randomized trial to assess the overall effectiveness of DKT to improve the postoperative quality of life following laparoscopic colectomy.

Trial registration: UMIN-CTR (Japan), UMIN000023318. Registered on 25 July 2016.

Keywords: TJ-100, Laparoscopic colectomy, Postoperative gastrointestinal symptoms

\footnotetext{
* Correspondence: kkawada@kuhp.kyoto-u.ac.jp

Department of Surgery, Graduate School of Medicine, Kyoto University, 54

Shogoin- Kawara-cho, Sakyo-ku, Kyoto 606-8507, Japan
} 


\section{Background}

Postoperative paralytic ileus can be a difficult complication for both surgeons and patients. Causes and treatments have been discussed for more than two centuries, but have not yet been fully resolved [1]. The incidence of postoperative ileus following colectomy is reported to be at least $10 \%$, and is considered to be inevitable for patients undergoing abdominal surgeries [2]. Patients who suffer from postoperative ileus require additional treatments such as fasting, antibiotics, decompression tubes, or even surgical intervention for suspected bowel strangulation. Owing to this, hospital stays lengthen and medical costs can increase more than $15 \%$ [2]. Therefore, the prevention of postoperative ileus is crucial for surgeons.

Daikenchuto (TJ-100, DKT) is a traditional Japanese herbal medicine (Kampo). This medicine is a crude drug extract and consists of four active components including processed ginger, ginseng, Japanese zanthoxylum peel and koi (maltose powder). In Japan, DKT has been clinically used for the treatment of postoperative paralytic ileus [3]. Recently, some beneficial mechanisms of DKT to relieve paralytic ileus have been reported. DKT can suppress inflammation, increase intestinal blood flow, and accelerate bowel movements [4-7]. The antiinflammatory effect is derived from inhibited activity of cyclooxygenase- 2 and upregulation of endogenous adrenomedullin $[4,5]$. An increase in intestinal blood flow is due to upregulation of calcitonin gene-related peptide [6]. Accelerated bowel movement is due to adjustment of the contraction and relaxation of the intestine by release of acetylcholine, nitric oxide and other excitatory neurotransmitters [8]. Therefore, DKT is considered to be a potential drug to reduce postoperative paralytic ileus and improve postoperative quality of life for patients with colon cancer.

\section{Methods/design Objective}

The aim of this study is to assess the effect of DKT on postoperative gastrointestinal symptoms following laparoscopic colectomy in patients with left-sided colon cancer.

\section{Study design and setting}

This study will be conducted at Kyoto University Hospital in Japan, and the design is an open-label randomized controlled trial. This study is expected to span a period of 2 years. Perioperative procedures in both groups will be performed based on the clinical path for laparoscopic colectomy in Kyoto University Hospital. All patients will receive standard bowel preparation $(75 \mathrm{mg}$ sodium picosulfate and $12 \mathrm{mg}$ pursennid) and antibiotic prophylaxis (oral doses of $1 \mathrm{~g}$ kanamycin and $750 \mathrm{mg}$ metronidazole), and surgical procedures will be conducted by well-experienced, board-certified laparoscopic colorectal surgeons at our institution. Except for the administration of DKT, treatment protocols including perioperative management and surgical procedures will be congruent among the intervention group (DKT group) and the control group (non-DKT group).

\section{Endpoints}

The following primary endpoints will be evaluated in this clinical trial: (i) grade of abdominal pain determined using the numeric rating scale (NRS), (ii) grade of abdominal distention determined using the NRS, and (iii) quality of life determined using the Gastrointestinal Quality Life Index (GIQLI) [9]. NRS measurements of abdominal pain and distention will be taken prior to surgery and at postoperative days (POD) 1, 4, 7, 14 and 28. GIQLI will be taken before surgery and at POD 14 and 28.

The secondary endpoints are (i) postoperative nutritional status (Onodera's prognostic nutritional index (PNI) [10] and Controlling Nutritional Status score (CONUT score) [11]), (ii) time to initial flatus, (iii) time to initial defecation, (iv) bowel gas volume measured using analysis software, (v) character of stool (Bristol Stool Form Scale [12]), (vi) defecation frequency per day, (vii) postoperative complications (Clavien-Dindo classification [13]), (viii) duration of postoperative hospital stay, and (ix) metabolomics analysis of metabolites in stool and blood using gas chromatography-tandem mass spectrometry (GC/MS/MS) and liquid chromatography-tandem mass spectrometry (LC/MS/MS) (Fig. 1).

\section{Eligibility criteria}

The eligible patients must satisfy the criteria defined below. Potential participants who meet the inclusion criteria will be recruited at outpatient clinics or during preoperative visits before surgery.

Inclusion criteria:

1. Patients with left-sided colon cancer (including rectosigmoid cancer) who are scheduled to undergo laparoscopic surgery

2. Clinical stage I, II and III

3. Patients who suffer from abdominal pain and distention at POD 1 (NRS score $\geq 1$ )

4. The European Cooperative Oncology Group (ECOG) performance status of 2 or less.

5. Patients aged 20 years and older at registration

6. Patients who can take medications orally

7. Written informed consent provided to participate in the study 


\begin{tabular}{|c|c|c|c|c|c|c|c|c|}
\hline \multirow[b]{3}{*}{ TIMEPOINT } & \multicolumn{8}{|c|}{ STUDY PERIOD } \\
\hline & \multirow{2}{*}{$\begin{array}{c}\text { Enrolment } \\
\text { Day1 }\end{array}$} & \multirow{2}{*}{$\begin{array}{c}\text { Allocation } \\
\text { Day1 }\end{array}$} & \multicolumn{5}{|c|}{ Post-allocation (Days) } & \multirow{2}{*}{$\frac{\text { Close-out }}{t_{x}}$} \\
\hline & & & 2 & 4 & 7 & 14 & 28 & \\
\hline \multicolumn{9}{|l|}{ ENROLMENT: } \\
\hline Eligibility screen & $x$ & & & & & & & \\
\hline Informed consent & $x$ & & & & & & & \\
\hline Allocation & & $\mathrm{X}$ & & & & & & \\
\hline \multicolumn{9}{|l|}{ INTERVENTIONS: } \\
\hline \multicolumn{9}{|l|}{ [Intervention] } \\
\hline \multicolumn{9}{|l|}{ [Control] } \\
\hline \multicolumn{9}{|l|}{ ASSESSMENTS: } \\
\hline Abdominal pain & $\mathrm{X}$ & $x$ & & $\mathrm{X}$ & $x$ & $x$ & $x$ & $\mathrm{X}$ \\
\hline $\begin{array}{c}\text { Abdominal } \\
\text { distention }\end{array}$ & & X & & $\mathrm{X}$ & $x$ & $x$ & $\mathrm{X}$ & $\mathrm{X}$ \\
\hline GLIQI & & 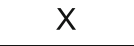 & & $\mathrm{X}$ & $X$ & $\mathrm{X}$ & $\mathrm{X}$ & $\mathrm{X}$ \\
\hline Nutritional status & & $x$ & & $\mathrm{X}$ & $x$ & $x$ & $\mathrm{x}$ & $\mathrm{X}$ \\
\hline Time to initial flatus & & & $\mapsto$ & & & & $\rightarrow$ & $x$ \\
\hline $\begin{array}{r}\text { Time to initial } \\
\text { defecation }\end{array}$ & & & $\leftarrow$ & & & & $\rightarrow$ & $x$ \\
\hline Bowel gas volume & & $x$ & & & $\mathrm{X}$ & & & $x$ \\
\hline Character of stool & & & & $x$ & $x$ & $x$ & $x$ & $x$ \\
\hline $\begin{array}{l}\text { Defecation } \\
\text { frequency }\end{array}$ & & & & $\mathrm{X}$ & $X$ & $x$ & $x$ & $\mathrm{X}$ \\
\hline Complication & & & $\leftarrow$ & & & & $\rightarrow$ & $x$ \\
\hline $\begin{array}{l}\text { Duration of } \\
\quad \text { Hospital stay }\end{array}$ & & & $\mapsto$ & & & & $\Rightarrow$ & $\mathrm{X}$ \\
\hline $\begin{array}{l}\text { Metabolomics } \\
\text { analysis } \\
\text { (blood / stool) }\end{array}$ & & & & $\mathrm{X}$ & $\mathrm{X}$ & $x$ & $x$ & $\mathrm{X}$ \\
\hline
\end{tabular}

Fig. 1 Schedule of enrolment, intervention, and assessments. GIQLI, Gastrointestinal Quality Life Index

Exclusion criteria:

1. Patients who have history of abdominal surgery or history of bowel obstruction

2. Patients with concomitant inflammatory bowel disease such as ulcerative colitis and Crohn's disease

3. Patients with concomitant endometriosis

4. Patients requiring emergency surgery

5. Patients who have been or will be treated by chemotherapy or radiotherapy

6. Patients with severe comorbidity such as cardiac disease, liver disease, pulmonary disease or renal disease

7. Patients who took Japanese herbal medicine (Kampo) up to 4 weeks prior to registration

8. Patients who took gastrointestinal prokinetic drugs, antipsychotic drugs or antidepressant drugs up to 4 weeks prior to registration
9. Patients with a history of a Kampo allergy in other formulations

10.Patients with hepatitis $\mathrm{B}$ or $\mathrm{C}$

11.Patients who are unable to take medications orally at POD 1

12.Patients who are unsuitable for study inclusion as determined by the investigator (e.g., those with severe dementia)

\section{Registration}

Patients will be enrolled at POD 1 . An eligibility report form will be sent to the registration center (APO PLUS STATION Co., Ltd, Tokyo, Japan). Eligible patients will be centrally randomized to either the DKT group or non-DKT group at the registration center using a computer random number generator with the minimization method for TNM stage, tumor location, and age. Information on the necessary follow-up 
evaluations will then be sent from the registration center. A flow diagram of this study is shown in Fig. 2.

\section{Treatment methods}

\section{Intervention group: DKT group}

DKT $(5 \mathrm{~g})$ will be administered orally three times per day between POD 2 and POD 28. DKT manufactured by Tsumura \& Co (Tokyo, Japan) will be used. We will monitor adherence by checking the number of doses of DKT that are not administered.

\section{Control group: non-DKT group}

No additional medicine will be administered as a comparator.

\section{Prohibited or limited drugs}

Prohibited drugs will be drugs that are known to regulate intestinal movement (erythromycin, mosapride citrate, pantethine, panthenol, prostaglandin $\mathrm{F} 2 \alpha$ ), digestive enzyme preparations (diastase, pancreatin), anticholinergic drugs (atropine sulfate, scopolamine butylbromide), cathartic drugs (sodium picosulfate), antidiarrheal drugs (albumin tannate, aluminum silicate, dimethicone), antipsychotic drugs (diazepam, flunitrazepam), lactobacillus preparation (lactomin, lactic acid bacteriae, Clostridium butyriam), anti-Parkinsonian drugs, and contrast agent (gastrografin).

Limited drugs will be antiemetic drugs including metoclopramide and domperidone. Rescue drugs will be antidiarrheal drugs (magnesium oxides, sennoside), cathartic

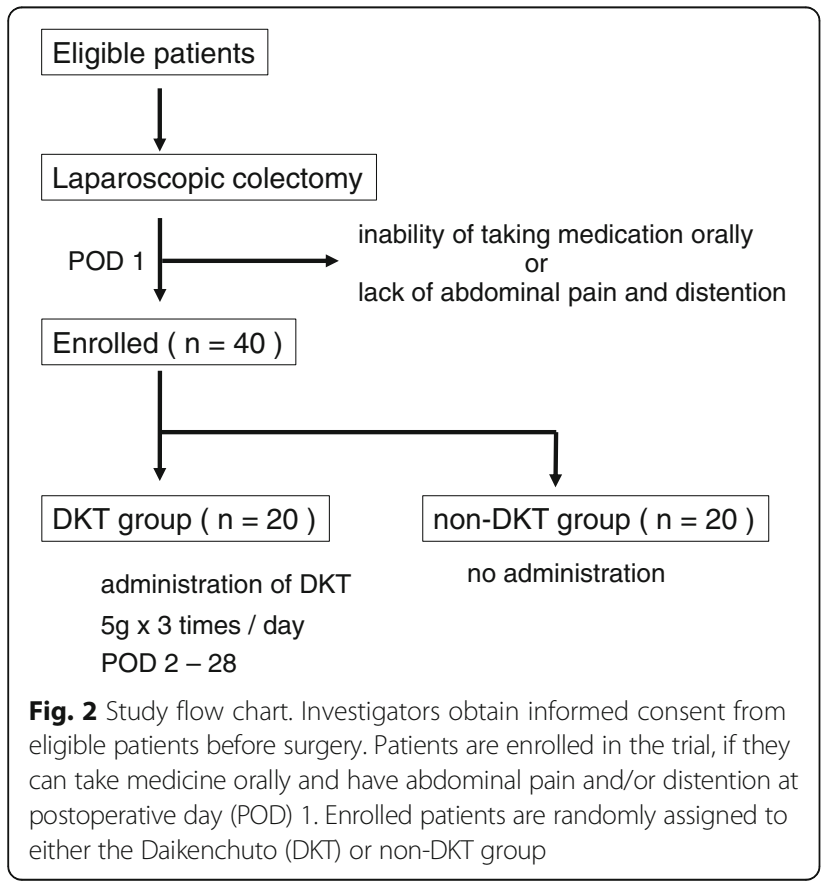

drugs (loperamide), and analgesic drugs (loxoprofen). If patients use the limited or rescue drugs, investigators will notate the dose and frequency.

\section{Criteria for discontinuing the protocol treatment}

If grade 3 postoperative adverse effects (Clavien-Dindo classification) are observed, the protocol treatment will be immediately discontinued.

\section{Data collection}

Prospective data about all patients including physical examination, laboratory data, perioperative clinical information and complications will be collected. Personal information including names and chart numbers will not be collected. Only the study code will be collected and managed separately. The collected data will remain confidential until the investigators analyze the data. The final dataset will be managed by the chief investigator (KK). After the completion of the study, the collected data will be encrypted and stored for 10 years and then discarded. Data collection, management, analysis, and interpretation of data will be performed by the registration center (APO PLUS STATION Co., Ltd).

\section{Sample size determination}

The sample size calculation is based on a primary endpoint, GIQLI. The GIQLI questionnaire containing 36 questions each with five response categories. The responses to questions are summed to give a numerical score (0-128 points). A previous report [9] indicates that the mean \pm standard deviation (SD) change in the GIQLI score between two measurements before and 2 weeks after laparoscopic cholecystectomy in 194 patients was 17 \pm 20.7 points. Therefore, the clinically significant mean difference in the GIQLI score is set at 20 with a SD of 20 . Using a power level of $80 \%$ and a two-sided significance level of 5\%, 32 patients (16 patients in each group) are required to show a difference between the DKT and nonDKT groups. Therefore, we determined that 40 patients (20 patients in each group) are needed to endure $20 \%$ dropout of patients from this trial.

\section{Statistical analysis}

All analyses will be performed under the intention-totreat principle. However, patients will be excluded from the analyses if they are proved to be ineligible after enrollment. Both primary and secondary endpoints will be compared between the DKT group and the non-DKT group. A two-tailed $P$ value less than 0.05 is considered statistically significant. For continuous variables, the $t$ test will be used when continuous variables follow a normal distribution, whereas the Wilcoxon rank-sum test will be used when continuous variables follow a non-normal 
distribution. For categorical variables, the chi-square test or Fisher's exact test will be used according to the cell count.

\section{Monitoring}

This trial is conducted based on Good Clinical Practice. Two people external to trial involvement will check the following at 6 months:

1. Informed consent and the data of primary endpoints for all patients

2. Eligibility criteria and hospital stay duration for a sample of 10 patients

\section{Study status}

This study is currently collecting data and there has not been any publication on the analysis of the data collected to date.

\section{Discussion}

This trial will elucidate the effect of DKT on postoperative gastrointestinal symptoms following laparoscopic colectomy in patients with colon cancer. We mainly focus on two important topics: postoperative quality of life and postoperative metabolites in stool and blood.

Although several trials have been conducted, the clinical effectiveness of DKT is still unclear. In previous randomized controlled trials, all primary endpoints were objective outcomes, such as duration to initial flatus and incidence of postoperative paralytic ileus [14-18]. Although some patients were reported to be improved by DKT administration, there has been no report where any subjective outcomes (i.e., postoperative quality of life) are set up as primary endpoints. Assessment of postoperative quality of life has been conducted as a secondary endpoint in some trials, but there was no significant difference between the intervention group and control group [15-18]. This study is the randomized controlled trial to investigate the effects of DKT on postoperative subjective outcomes (i.e., postoperative quality of life) following laparoscopic colectomy as primary endpoints. Moreover, exploratory metabolomics analysis of metabolites in stool and blood will be conducted in this trial. It was reported that DKT could influence the microbiome of stool through bacterial metabolism in animals' bowels $[19,20]$. However, there is no report to date investigating this in humans. Using metabolomics analysis of metabolites in stool and blood, we plan to examine the effect of the intestinal flora by DKT administration, which can lead to the elucidation of the mechanism of DKT in gastrointestinal symptoms and the discovery of the biomarker candidates.

Therefore, we designed this trial to evaluate the effect of DKT on postoperative quality of life and postoperative metabolites in patients with colon cancer undergoing laparoscopic colectomy. The study aims to guide a future full-scale pragmatic randomized trial to assess the overall effectiveness of DKT to improve the postoperative quality of life following laparoscopic colectomy. Methodological rigor, including random allocation and prospective trial registration is aimed at reducing the risk of bias. As a limitation of this study, the possibility of insufficient sample size, bias in the open-label design, and the possibility of unblinding of the assessors to outcome may result in performance bias.

\section{Trial status}

Recruitment of participants has been ongoing since September 2016.

\section{Additional file}

Additional file 1: SPIRIT 2013 checklist: recommended items to address in a clinical trial protocol and related documents. (DOC $123 \mathrm{~kb}$ )

\section{Abbreviations \\ CONUT: Controlling nutritional status; DKT: Daikenchuto; GIQLI: Gastrointestinal Quality Life Index; NRS: Numeric rating scale; PNI: Prognostic nutritional index; POD: Postoperative day; SD: Standard deviation}

\section{Acknowledgements}

Not applicable.

Funding

This study was supported by a research grant from Tsumura \& Co, Tokyo, Japan.

Availability of data and materials

Requested data for public purpose or research transparency will be provided on reasonable request (please contact the corresponding author).

\section{Authors' contributions}

$\mathrm{KK}$ and YS conceived the study. KK and $\mathrm{KH}$ initiated the study design and drafted the protocol. NH, TW, RT, MY, and YS contributed to the refinement of the study protocol. $\mathrm{NH}$ and $\mathrm{KK}$ prepared the first draft of the manuscript. $\mathrm{KH}, \mathrm{TW}, \mathrm{RT}, \mathrm{MY}$, and YS critically reviewed, revised and approved the final version. All authors read and approved the final manuscript.

\section{Ethics approval and consent to participate}

This protocol has been prepared in accordance with Standard Protocol Items: Recommendations for Interventional Trials (SPIRIT), a completed SPIRIT checklist is included as Additional file 1.

This study protocol has been conducted according to the Declaration of Helsinki, and was approved by the institutional review board of Kyoto University, Kyoto, Japan (C1112-1) and by the institutional review board of Tsumura \& Co, Tokyo, Japan (1502). Participation in the trial never influences the therapeutic strategy and treatment burden. Written informed consent will be obtained from all participants by the investigator.

\section{Consent for publication}

Not applicable.

Competing interests

The authors declare that they have no competing interests. 


\section{Publisher's Note}

Springer Nature remains neutral with regard to jurisdictional claims in published maps and institutional affiliations.

Received: 8 April 2017 Accepted: 2 November 2017

Published online: 21 November 2017

\section{References}

1. Augestad KM, Delaney CP. Postoperative ileus: impact of pharmacological treatment, laparoscopic surgery and enhanced recovery pathways. World J Gastroenterol. 2010;16:2067-74.

2. lyer S, Saunders WB, Stemkowski S. Economic burden of postoperative ileus associated with colectomy in the United States. J Manag Care Pharm. 2009; 15:485-94.

3. Itoh T, Yamakawa J, Mai M, Yamaguchi N, Kanda T. The effect of the herbal medicine dai-kenchu-to on post-operative ileus. J Int Med Res. 2002;30:428-32.

4. Hayakawa T, Kase Y, Saito K, Hashimoto K, Ishige A, Komatsu Y, et al. Effects of Dai-kenchu-to on intestinal obstruction following laparotomy. J Smooth Muscle Res. 1999;35:47-54.

5. Kono T, Omiya Y, Hira Y, Kaneko A, Chiba S, Suzuki T, et al. Daikenchuto (TU-100) ameliorates colon microvascular dysfunction via endogenous adrenomedullin in Crohn's disease rat model. J Gastroenterol. 2011;46:1187-96.

6. Kono $\mathrm{T}$, Koseki T, Chiba S, Ebisawa Y, Chisato N, Iwamoto J, et al. Colonic vascular conductance increased by Daikenchuto via calcitonin gene-related peptide and receptor-activity modifying protein 1. J Surg Res. 2008;150:78-84

7. Fukuda H, Chen C, Mantyh C, Ludwig K, Pappas TN, Takahashi T. The herbal medicine, Dai-Kenchu-to, accelerates delayed gastrointestinal transit after the operation in rats. J Surg Res. 2006;131:290-5.

8. Kito $Y$, Suzuki $H$. Effects of Dai-kenchu-to on spontaneous activity in the mouse small intestine. J Smooth Muscle Res. 2006;42:189-201.

9. Eypasch E, Williams Jl, Wood-Dauphinee S, Ure BM, Schmülling C Neugebauer E, et al. Gastrointestinal Quality of Life Index: development, validation and application of a new instrument. Br J Surg. 1995:82:216-22.

10. Onodera T, Goseki N, Kosaki G. Prognostic nutritional index in gastrointestinal surgery of malnourished cancer patients. Nihon Geka Gakkai Zasshi. 1984;85:1001-5.

11. Ignacio de Ulibarri J, Gonzalez-Madrono A, de Villar NG, González P, González B, Mancha A, et al. CONUT: a tool for controlling nutritional status. First validation in a hospital population. Nutr Hosp. 2005;20:38-45.

12. Lewis SJ, Heaton KW. Stool form scale as a useful guide to intestinal transit time. Scand J Gastroenterol. 1997;32:920-4.

13. Clavien PA, Barkun J, de Oliveira ML, Vauthey JN, Dindo D, Schulick RD, et al. The Clavien-Dindo classification of surgical complications: five-year experience. Ann Surg. 2009;250:187-96.

14. Shimada M, Morine $Y$, Nagano $H$, Hatano E, Kaiho T, Miyazaki M, et al. Effect of TU-100, a traditional Japanese medicine, administered after hepatic resection in patients with liver cancer: a multi-center, phase III trial (JFMC401001). Int J Clin Oncol. 2015;20:95-104.

15. Akamaru Y, Takahashi T, Nishida T, Omori T, Nishikawa K, Mikata S, et al. Effects of daikenchuto, a Japanese herb, on intestinal motility after total gastrectomy: a prospective randomized trial. J Gastrointest Surg. 2015;19:467-72.

16. Katsuno H, Maeda K, Kaiho T, Kunieda K, Funahashi K, Sakamoto J, et al. Clinical efficacy of Daikenchuto for gastrointestinal dysfunction following colon surgery: a randomized, double-blind, multicenter, placebo-controlled study (JFMC39-0902). Jpn J Clin Oncol. 2015;45:650-6.

17. Yoshikawa K, Shimada M, Wakabayashi G, Ishida K, Kaiho T, Kitagawa Y, et al Effect of daikenchuto, a traditional Japanese herbal medicine, after total gastrectomy for gastric cancer: a multicenter, randomized, double-blind, placebo-controlled, Phase II Trial. J Am Coll Surg. 2015;221:571-8.

18. Okada K, Kawai M, Hirono S, Fujii T, Kodera Y, Sho M, et al. Evaluation of the efficacy of daikenchuto (TJ -100) for the prevention of paralytic ileus after pancreaticoduodenectomy: a multicenter, double-blind, randomized, placebo-controlled trial. Surgery. 2016;159:1333-41.

19. Yoshikawa K, Shimada M, Kuwahara T, Hirakawa H, Kurita N, Sato H, et al. Effect of Kampo medicine "Dai-kenchu-to" on microbiome in the intestine of the rats with fast stress. J Med Invest. 2013;60:221-7.

20. Hasebe T, Ueno N, Musch MW, Nadimpalli A, Kaneko A, Kaifuchi N, et al. Daikenchuto (TU-100) shapes gut microbiota architecture and increases the production of ginsenoside metabolite compound K. Pharmacol Res Perspect. 2016;4:1-10.

\section{Submit your next manuscript to BioMed Central and we will help you at every step:}

- We accept pre-submission inquiries

- Our selector tool helps you to find the most relevant journal

- We provide round the clock customer support

- Convenient online submission

- Thorough peer review

- Inclusion in PubMed and all major indexing services

- Maximum visibility for your research

Submit your manuscript at www.biomedcentral.com/submit 